\title{
Palliativmedizin in der Pneumologie
}

\author{
Palliative Care in Respiratory Medicine
}

Autoren

Institute
H. Magnussen ${ }^{1}$, D. F. Heigener ${ }^{1}$, C. Bausewein ${ }^{2}$, M. Thomas ${ }^{3}$, C. Eschbach ${ }^{4}$, W. Schütte ${ }^{5}$, T. Blankenburg ${ }^{5}$, M. Steins ${ }^{3}$, H. Bischoff ${ }^{3}$, B. Schucher ${ }^{1}$, S. Rosseau ${ }^{6}$, A. Schütz ${ }^{7}$, J. Geiseler ${ }^{8}$, S. Kampf ${ }^{9}$, O. Karg ${ }^{8}$

für die Arbeitsgemeinschaft Palliativmedizin der Pneumologie

Die Institutsangaben sind am Ende des Beitrags gelistet. eingereicht 11.2.2009

akzeptiert nach Revision 6. 3.2009

\section{Bibliografie}

DOI $10.1055 / \mathrm{s}-0029-1214538$

Pneumologie 2009; 63: 289-295

(c) Georg Thieme Verlag KG

Stuttgart · New York

ISSN 0934-8387

\section{Korrespondenzadresse}

Prof. Dr. H. Magnussen

Ärztlicher Direktor

Krankenhaus Großhansdorf

Zentrum für Pneumologie und

Thoraxchirurgie

Lehrstuhl für Innere Medizin-

Pneumologie

Sitz Großhansdorf

Universität Lübeck

Wöhrendamm 80

22927 Großhansdorf

h.magnussen@kh-

grosshansdorf.de

\section{Zusammenfassung \\ $\nabla$}

Die palliativmedizinische Versorgung sollte Bestandteil der Pneumologie sein, da erstens viele pneumologische Krankheitsbilder - neben den thorakalen Tumoren - in ihrem Verlauf palliativmedizinischer Betreuung bedürfen und zweitens die Dyspnoe auch als Symptom fortgeschrittener, primär extrapulmonaler Erkrankungen der Expertise des Pneumologen bedarf. In dieser Arbeit werden die häufigsten Symptome fortgeschrittener pneumologischer Erkrankungen sowie ihre Behandlung dargestellt. Daneben werden die Versorgungsstrukturen in Deutschland dargestellt.

\section{Einleitung \\ $\nabla$}

Die Palliativmedizin ist die Fortführung der optimalen Therapie des Patienten mit geändertem Therapieziel: Statt auf Heilung liegt der Fokus nun auf Symptomlinderung und ganzheitlicher Betreuung des Patienten unter Einbeziehung seiner Bezugspersonen. Die Palliativmedizin sollte keineswegs ausschließlich in der Finalphase, sondern schon in früheren Stadien praktiziert werden [1].

Die Palliativmedizin wird gegenwärtig vornehmlich mit der Betreuung onkologischer Erkrankungen verbunden. Es ist jedoch erforderlich, Patienten mit inkurablen, nicht malignen Erkrankungen in die palliativmedizinische Versorgung zu integrieren. In der Pneumologie leiden zahlreiche Patienten mit nicht malignen Erkrankungen unter chronischen Verläufen, die einer Betreuung nach palliativmedizinischen Grundsätzen bedürfen. Die chronisch obstruktiven Lungen- und Atemwegserkrankungen (COPD) zählen zu den häufigsten internistischen Erkrankungen, die vorzeitig zum Tode führen. Patienten mit Lungengerüsterkrankungen bedürfen in den fortgeschrittenen Krankheitsstadien ebenso einer palliativmedizi-

\section{Abstract \\ $\nabla$}

Palliative care should be part of respiratory medicine for two reasons: First, many respiratory diseases - besides thoracic tumours - need palliative care in the late stages of the disease. Second, dyspnoea is a common symptom in advanced, primary extrapulmonary diseases and the knowledge of respiratory specialists can be beneficial in the treatment of this symptom. In this paper we describe frequent symptoms of advanced pulmonary diseases and their treatment. Moreover, we focus on the structure of palliative care in Germany.

nischen Versorgung. Patienten mit neuromuskulären (z.B. Amyotrophe Lateralsklerose) oder muskuloskeletalen Erkrankungen (z.B. Kyphoskoliose) leiden unter Symptomen, die durch eine nicht invasive Beatmung gelindert werden können. Der Pneumologe betreut daher nicht nur eine Vielzahl von Patienten mit Lungen- und Atemwegserkrankungen, sondern verfügt auch über die Expertise, die pulmonalen Konsequenzen extrapulmonaler Erkrankungen zu behandeln.

Der Einsatz der nicht invasiven Beatmung hat durch die bedeutsame Abnahme beatmungsassoziierter Komplikationen, die mit einer invasiven Beatmung verknüpft waren, einen Siegeszug in der pneumologischen Intensivmedizin angetreten. Die nicht invasive Beatmung im häuslichen und stationären Umfeld ist heute ein Bestandteil der palliativen Versorgung. Der Einsatz derartiger Maßnahmen bedarf jedoch der sorgsamen Abwägung des Nutzens für die Lebensqualität des Patienten [2]. Es bedarf noch erheblicher Anstrengungen, den Dialog zwischen den Patienten und ihren Angehörigen sowie dem betreuenden ärztlichen und nicht ärztlichen Personal zu verbessern, um den Einsatz der intensivmedizinischen 
Maßnahmen unter den Gesichtspunkten der Palliativmedizin zu verbessern.

Das Ziel der Arbeitsgemeinschaft Palliativmedizin in der Pneumologie ist die Betreuung von Patienten mit thorakalen Tumoren und nicht malignen pneumologischen Krankheitsbildern zu gewährleisten und durch geeignete Versorgungsstrukturen zu verbessern.

In diesem Beitrag werden die palliativmedizinischen Möglichkeiten bei den verschiedenen pneumologischen Krankheitsbildern zusammengefasst. Wir haben dabei ein besonderes Gewicht auf die therapeutischen Möglichkeiten bei der Dyspnoe sowie der Behandlung von Schmerzen, Kachexie und Fatigue gelegt.

Dieses Papier soll einen Dialog eröffnen, um durch pneumologische Kompetenz die Palliativmedizin zu bereichern.

\section{Maligne Erkrankungen der Thoraxorgane}

Die Diagnostik und die Therapie thorakaler maligner Erkrankungen bedarf in der Regel interdisziplinärer Zusammenarbeit von Pneumologen, Thoraxchirurgen und Strahlentherapeuten sowie der Einbindung von Onkologen, Palliativmedizinern und anderen Berufsgruppen. Aus epidemiologischen Arbeiten ist bekannt, dass nur dieses Vorgehen den Patienten den Zugang zu den aktuellen Behandlungsstandards sichert und dass Patienten mit diesen Erkrankungen, die in Zentren betreut werden, signifikant länger überleben. Andererseits zeigt die gleiche Studie auch, dass nur 75\% aller Patienten mit malignen thorakalen Erkrankungen in Zentren vorgestellt werden [3].

Während die aktive onkologische Therapie in Zentren den Patienten Vorteile bringt, muss die palliativmedizinische Versorgung im gewohnten und gewünschten Umfeld des Patienten erfolgen. Der patientenzentrierte Ansatz der Palliativmedizin wird regional orientiert nur unter der Einbeziehung der niedergelassenen Pneumologen, Hausärzte, Palliativmediziner und Onkologen sowie aller notwendigen nicht ärztlichen Berufsgruppen erfolgreich sein [4].

\section{Dyspnoe bei Thoraxtumoren}

Dyspnoe ist ein häufiges Symptom von Patienten mit thorakalen Tumoren, vor allem dann, wenn Begleiterkrankungen wie eine COPD vorliegen. Der Begriff „Dyspnoe“ wird von den Patienten selbst nicht verwendet. Diese benutzen eher Begriffe wie „Lufthunger“, „Druck auf der Brust“ oder „Kurzatmigkeit“, die manchmal Rückschlüsse auf die pathophysiologischen Mechanismen der Dyspnoe erlauben [5]. Die Quantifizierung kann anhand von eindimensionalen Skalen, wie z.B. der Borg-Skala oder einer visuellen Analogskala, erfolgen.

Das primäre Ziel jeder therapeutischen Maßnahme sollte die Beseitigung der Ursache der Atemnot sein, z. B. die Punktion eines Pleura- oder Perikardergusses oder die bronchologische Intervention bei Stenosen der zentralen Atemwege.

Die Therapie der Dyspnoe bei Patienten mit malignen Erkrankungen der Thoraxorgane weist vor allem im Endstadium der Erkrankung die Besonderheit auf, dass eine nicht invasive oder gar invasive Beatmung aufgrund der Prognose meist nicht in Betracht kommt. Diese Form der Therapie wird in dem korrespondierenden Kapitel über die nicht malignen Erkrankungen diskutiert.

Viele der Patienten mit Bronchialkarzinom haben trotz Atemnot keinen Nachweis einer Hypoxämie. Der Einsatz von Sauerstoff bei diesen Patienten ist daher auch nicht indiziert [6], der manch-
Tab. 1 Medikamente bei Dyspnoe.

\begin{tabular}{|c|c|c|}
\hline Medikament & Dosierung & $\begin{array}{l}\text { Evidenz- } \\
\text { grad }\end{array}$ \\
\hline Morphinsulfat & $\begin{array}{l}5-20 \text { mg s. c. alle } 6-8 \text { Stunden } \\
\text { bei opioid-naiven Patienten } \\
20-25 \% \text { Dosissteigerung } \\
\text { bei vorbehandelten Patienten } \\
10-30 \text { mg retardiert alle } \\
12 \text { Stunden }\end{array}$ & B \\
\hline Lorazepam & $\begin{array}{l}1-2,5 \text { mg buccal alle } \\
6-8 \text { Stunden }\end{array}$ & C \\
\hline Midazolam & $\begin{array}{l}2,5-5 \mathrm{mg} \text { Bolus i. v. } \\
\text { bis } 20 \mathrm{mg} \text { tgl. kontinuierlich } \\
\text { als Perfusor }\end{array}$ & C \\
\hline Promethazin & - 20-50 mg p.o. alle 8 Stunden & C \\
\hline Levomepromazin & - 10 - 20 mg alle 8 Stunden & C \\
\hline
\end{tabular}

mal vom Patienten empfundene positive Effekt mag auf die entstehende Luftströmung zurück zu führen sein. Diese kann auch durch Handventilatoren erzeugt werden, welche wir in unserem Zentrum vor allem bei anfallsartiger Luftnot manchmal empfehlen.

Studien zu nicht pharmakologischen Ansätzen zur Therapie der Dyspnoe wurden kürzlich in einem Cochrane-Review systematisch erfasst [7]: Atemtraining, Gehhilfen, neuromuskuläre Stimulation und Vibrationsmassage der Brustwand zeigten hier einen möglichen positiven Effekt. Der Effekt von Handventilatoren wurde nur in zwei Studien untersucht und konnte nicht definitiv bewertet werden. Eine große Einschränkung dieses Reviews ist die Tatsache, dass die meisten Studien mit COPD-Patienten durchgeführt wurden. Es ist unklar, inwieweit die Ergebnisse auf Tumorpatienten übertragen werden können.

Zur medikamentösen Therapie der Dyspnoe stehen im Wesentlichen zwei Substanzgruppen zur Verfügung: Opioide und Benzodiazepine. Opioide zeigen einen positiven Effekt auf die Dyspnoe, wenn sie enteral oder parenteral zugeführt werden, inhalativ haben sie keinen gesicherten Effekt [8]. Die optimale Dosis ist bisher nicht bekannt. Bei opioid-naiven Patienten sollte man mit $5 \mathrm{mg}$ nicht retardiertem Morphinsulfat s.c. beginnen. Vortherapierte Patienten sollten eine Erhöhung ihrer Opioiddosis von 20-25\% erhalten ( $\bullet$ Tab. 1 ).

Aufgrund ihrer anxiolytischen Eigenschaften werden Benzodiazepine, wie Lorazepam (z. B. Tavor ${ }^{\circledR}$ ) oder Diazepam (z. B. Valium $^{\circledR}$ ), regelmäßig bei Patienten mit Atemnot verschrieben. Die Evidenz für den Einsatz von diesen Medikamenten bei Atemnot ist allerdings gering. Das Abhängigkeitspotenzial der Benzodiazepine steht bei fortgeschrittenen Tumorerkrankungen sicher im Hintergrund. Trotzdem sollte die lange Wirkdauer und Akkumulationsgefahr bei diesen Medikamenten bedacht werden. Aus diesem Grund ist Lorazepam (0,5-1 mg) gegenüber Diazepam zu bevorzugen. In der Terminalphase, wenn eine stärkere Sedierung u.U. notwendig ist, kann Midazolam (z. B. Dormicum ${ }^{\circledR}$ ) subkutan, entweder als Bolus 2,5-5 mg oder kontinuierlich 5$20 \mathrm{mg} / 24 \mathrm{~h}$ verabreicht werden. Benzodiazepine können grundsätzlich auch gut mit Morphin kombiniert werden [9] ( $\bullet$ Tab. 1). Auch Phenothiazine wie Promethazin (z. B. Atosil ${ }^{\circledR}$ ) oder Levomepromazin (z.B. Neurocil ${ }^{\circledR}$ ) werden alternativ zu Benzodiazepinen bei Atemnot eingesetzt. Auch hier ist die Evidenzlage eher spärlich. Phenothiazine wirken wie Benzodiazepine anxiolytisch, haben aber kein Abhängigkeitspotenzial ( $\bullet$ Tab. 1). Kleinere Studien zeigten eine positive Wirkung von Serotoninwiederaufnah- 
me-Hemmern (SSRI) wie z. B. Sertralin $\left(\right.$ Zoloft $\left.^{\circledR}\right)$ bei der Behandlung von Atemnot. Es ist allerdings nicht klar, ob dies ein direkter Effekt auf Atemnot oder auf eine gleichzeitige Angststörung/Depression war. Leider gab es keine weiteren Studien in den letzten Jahren, sodass der Einsatz von SSRI zur Therapie der Atemnot nicht eindeutig empfohlen werden kann [10]. Allerdings sollte bei allen Patienten mit Atemnot immer eine Angststörung und/ oder Depression bedacht und gegebenenfalls entsprechend behandelt werden.

\section{Therapie von Schmerzen bei Thoraxtumoren}

Schmerz ist einerseits ein körperlich empfundenes Symptom, andererseits ist es durch die psychische Wahrnehmung und individuelle Verarbeitung geprägt, sodass Charakter und Ausmaß nur vom Patienten selbst beurteilt werden können. Bei onkologischen Patienten wird der überwiegende Teil der Schmerzen direkt vom Tumor ausgelöst (60-90\%). Ein geringerer Anteil ist tumorassoziiert (5-20\%), therapiebedingt (10-25\%) oder gänzlich tumorunabhängig (3-10\%). Zu unterscheiden sind Nozizeptorschmerzen, die durch direkte Reizung von Schmerzrezeptoren ausgelöst werden, und neuropathische Schmerzen, welche durch Irritation und Kompression peripherer Nerven entstehen.

Aufgrund der Subjektivität der Wahrnehmung ist die Erfassung von Schmerzart und Schmerzintensität zusätzlich zu Anamnese und Vorbefunden von zentraler Bedeutung für das Erreichen einer ausreichenden Schmerzeinstellung und -kontrolle. Vor allem für die Einstellphase der Schmerzmedikation werden hierzu verbale oder numerische Rating-Skalen bzw. die visuelle AnalogSkala angewandt.

\section{Empfehlungen zur medikamentösen Schmerztherapie}

Die medikamentöse Therapie erfolgt gemäß den Leitlinien zur „Medikamentösen Schmerztherapie“ und basiert auf dem Einsatz von Nicht-Opioid-Analgetika der WHO-Stufe I (vor allem Metamizol, Ibuprofen, Diclophenac, Celecoxib) sowie bei nicht ausreichendem Ansprechen auf der Kombination mit OpioidAnalgetika der WHO-Stufe II (Tramadol, Tilidin) oder III (Morphin, Hydromorphon, Oxycodon, Fentanyl, Buprenorphin, L-Methadon). Für das Erreichen einer raschen analgetischen Wirkung ist zu berücksichtigen, dass im Fall von starken Tumorschmerzen nur die WHO-Stufe III eine ausreichende Effektivität verspricht. Die Schmerztherapie sollte bei Anwendung von Opioiden zu Beginn der medikamentösen Einstellung mit einem oral applizierbaren Präparat eingeleitet und titriert werden. Mit Abschluss der Einstellphase sollte für die ambulante und längerfristige Opioidgabe auf oral verfügbare Retardpräparate zurückgegriffen werden. Transdermal anwendbare Wirkstoffe, wie Fentanyl oder Buprenorphin, stellen eine Alternative für Patienten mit einem stabilen Schmerzniveau oder mit Schluckstörungen bzw. Problemen bei oraler Applikation dar. Bei Durchbruchschmerzen ist auf die ausreichende Bereitstellung eines schnell wirksamen Opioids als Bedarfsmedikation zusätzlich zur Basistherapie zu achten. Ziel bleibt es aber, Analgetika nach einem festen Zeitplan so zu applizieren, dass eine überlappende Wirkung ohne Auftreten von intermittierenden Schmerzattacken erreicht wird. Die Wirkstoff-spezifischen Anwendungsbeschränkungen und Kontraindikationen (Ulcerogenität, Nephro- u. Hepatotoxizität) sind besonders bei der Wahl der geeigneten Nicht-Opioide zu berücksichtigen. Bei Opioiden ist die adjuvante Medikation zu beachten. Diese umfasst eine kontinuierliche Obstipationsprophylaxe (z. B. Macrogol) sowie in der Einstellphase eine antiemetische Begleitmedikation (Metoclopramid, Haloperidol) [11].
Ko-Analgetika (Corticosteroide, Amitryptilin, Pregabalin, Gabapentin, Scopolamin, Carbamazepin, Bisphosphonate) haben in Ergänzung zur Schmerzbehandlung einen therapeutischen Stellenwert in Abhängigkeit von der jeweiligen Schmerzart, besonders bei neuropathischen Schmerzen [12].

\section{Empfehlungen zu interventionellen Schmerztherapien}

Tumorschmerzen, insbesondere durch lokalisierbare Tumore ausgelöst, lassen sich neben der medikamentösen Therapie durch lokale Strahlentherapie behandeln. Dies trifft vor allem für solitäre pleurale oder ossäre Metastasen zu. Im Falle multipler, schmerzhafter Knochenmetastasen kann auch eine Radionuklidtherapie (Strontium-89, Samarium-153) in Betracht gezogen werden für Patienten, bei denen eine medikamentöse Schmerztherapie nicht ausreichend oder eine lokale Radiatio nicht sinnvoll ist. Dieses Vorgehen wird aber in der klinischen Praxis nur für wenige Patienten in Frage kommen.

Ebenso haben Nervenblockaden oder Neurolysen angesichts der medikamentösen Schmerztherapien nur noch einen geringen klinischen Stellenwert und werden allenfalls in Einzelfällen nach Versagen medikamentöser oder strahlentherapeutischer Maßnahmen angewandt.

Auch die Elektrostimulation, von denen die transkutane elektrische Nervenstimulation (TENS) aufgrund der einfachen Handhabung das bekannteste Verfahren darstellt, hat bei tumorbedingten Schmerzen eine untergeordnete Bedeutung. Mögliche Einsatzgebiete stellen neuropathische Schmerzareale dar.

Schließlich können thorakale Schmerzzustände bei Palliativpatienten neben dem Tumorgeschehen auch auf einer eingeschränkten Funktion von Lunge und Atemhilfsmuskulatur beruhen. Hier ist der Einsatz von physiotherapeutischen Maßnahmen, wie Atemtherapie, und physikalischen Therapieansätzen, wie Inhalationsbehandlung und Massagen, sinnvoll $[13,14]$.

\section{Fatigue und Kachexie bei Thoraxtumoren}

Fatigue ist definiert als das permanente subjektive Gefühl der physischen, emotionalen und/oder kognitiven Erschöpfung, welche unproportional zur gegenwärtigen Tätigkeit ist und mit den Aktivitäten des täglichen Lebens interferiert. Es wird als sehr belastend empfunden und ist nicht selten das Hauptsymptom bei thorakalen Tumoren.

Die Ursachen sind vielfältig, teils Folge der Krankheit, teils Folge der Therapie. Einige sind behandelbar, wie z.B. Hyperkalzämie, Hypothyreose oder Anämie. Diese sollten vor einer symptomatischen Therapie ausgeschlossen sein bzw. kausal behandelt werden.

Die symptomatische Behandlung der Fatigue ist schwierig, zumal der Pathomechanismus nicht ergründet ist. Diskutiert werden ein direkter zentralnervöser Effekt von Therapeutika (Chemound/oder Strahlentherapie), der Verlust von Muskelmasse, pathologischer Energiemetabolismus in den Muskeln sowie systemisch-entzündliche Ursachen.

Das Amphetaminderivat Methylphenydat hat in einer Metaanalyse einen - sehr moderaten - positiven Effekt auf die Symptomatik gezeigt [15]. Das ursprünglich bei der Narkolepsie eingesetzte Modafinil ist vor allem bei schwerer Fatigue hilfreich, wie eine kürzlich als Abstract veröffentlichte Studie gezeigt hat [16]. Beide Präparate haben keinen Einfluss auf eine depressive Stimmungslage. Dies verdeutlicht, wie wichtig es ist, Fatigue von einer Depression zu differenzieren. Antidepressiva spielen dementsprechend nur dann eine Rolle in der Therapie, wenn der Er- 
schöpfungszustand Ausdruck einer Depression ist oder beide Krankheitsbilder gemeinsam vorliegen.

Der Einsatz von Steroiden ist wissenschaftlich nicht gut belegt, in der Praxis zeigen sich jedoch allenthalben Erfolge. Die Dauertherapie ist jedoch mit den bekannten Risiken behaftet, diese relativieren sich in Anbetracht der Lebenserwartung der Patienten.

Erythropoetin-Analoga (EPO's) wurden früher häufig zur Therapie von Tumoranämien eingesetzt und haben eine nachweisbare positive Wirkung auf die Fatigue. Da mehrere Studien jedoch zeigen, dass der Einsatz von EPO's die Rate von thrombembolischen Komplikationen erhöht und eventuell sogar die Tumorprogression fördert [17], sollte sie nur noch bei chemotherapie-induzierter Anämie eingesetzt werden. Aber auch hier ist ihr Einsatz wegen der Wirklatenz von Wochen aus unserer Sicht sehr zweifelhaft.

Neben pharmakologischen Ansätzen gibt es auch andere Therapiemöglichkeiten, hauptsächlich Verhaltensmodifikationen des Patienten. Am wichtigsten ist körperliches Training, welches die Symptomatik nachweislich lindert [18]. Auch eine gute Schlafhygiene und Verhaltenstherapie können eingesetzt werden.

Die Fatigue ist häufig vergesellschaftet mit einem weiteren häufigen Symptom bei Patienten mit Thoraxtumoren: der Kachexie. Diese ist definiert als ein ungewollter Gewichtsverlust, der neben Speicherfett auch das Strukturfett des Menschen betrifft (Wangen, Augenhöhlen). Auf weitere Einzelheiten wird im Kapitel „pulmonale Kachexie“ eingegangen, da sich die Therapie bei malignen und nicht malignen Erkrankungen nicht unterscheidet.

\section{Nicht maligne Erkrankungen der Thoraxorgane} $\nabla$

\section{Therapie der Dyspnoe}

Bezüglich der medikamentösen Therapie der Dyspnoe sei auf das korrespondierende Kapitel „Dyspnoe bei Thoraxtumoren“ und die aktuelle Leitlinie zur Langzeit-Sauerstofftherapie verwiesen [19].

\section{Nicht invasive Beatmung in der Palliativmedizin}

Die nicht invasive Beatmung (NIV) hat sich als effektive therapeutische Maßnahme bei einer ganzen Reihe von unterschiedlichen Erkrankungsbildern etabliert. Dies betrifft sowohl primär pneumologische als auch nicht pneumologische Erkrankungsentitäten wie z.B. neuromuskuläre Erkrankungen. NIV wird sowohl bei der akuten als auch chronischen respiratorischen Insuffizienz eingesetzt, einerseits als effektive lebenserhaltende und -verlängernde Maßnahme, in anderen Fällen aber auch als überwiegend symptombezogene, Beschwerden lindernde Therapie mit dem Ziel der Verbesserung der gesundheitsbezogenen Lebensqualität [20] und nur sekundär lebensverlängernd. Da sich diese beiden Therapieeffekte nicht klar voneinander trennen lassen, muss der behandelnde Arzt bei Einleitung einer NIV zusammen mit dem Patienten ein Therapieziel formulieren.

\section{NIV bei chronisch respiratorischer Insuffizienz}

Bei der chronisch ventilatorischen Insuffizienz sind palliativmedizinische Aspekte von erheblicher Bedeutung. Insbesondere bei weit fortgeschrittenen Erkrankungsstadien bei Patienten mit COPD oder Amyotropher Lateralsklerose steht oft nicht primär die Lebensverlängerung, sondern die Symptomlinderung und die Verbesserung der Lebensqualität im Vordergrund. Diese konnte in einer Reihe von Studien für den überwiegenden Anteil der mit einer nicht invasiven außerklinischen Beatmung behan- delten Patientengruppen belegt werden [21]. Bei Patienten mit COPD ist neben einer Verminderung der Dyspnoe auch eine Verbesserung der körperlichen Belastbarkeit belegt [22]. Ein weiterer für die Patienten wichtiger Aspekt ist die Reduktion der Häufigkeit und Dauer von stationären Krankenhausaufenthalten [23].

\section{Pulmonale Kachexie bei nicht malignen Erkrankungen der Thoraxorgane}

Gewichtsverlust ist eine häufige Komplikation chronisch pulmonaler Erkrankungen, z.B. entwickeln ca. 25\% der Patienten mit COPD eine Kachexie. Für andere pulmonale Erkrankungen - z. B. Lungenfibrose - gibt es keine verlässlichen Zahlen.

Kachexie ist schlecht definiert, früher wurde ein Body-Mass-Index unter $21 \mathrm{~kg} \mathrm{~m}^{2}$ als Kachexie eingestuft. Hierbei wird aber die Körperzusammensetzung, v.a. der Fettanteil, nicht berücksichtigt. Deshalb sollte Kachexie als fettfreie Masse oder lean body mass index $<16 \mathrm{~kg} \mathrm{~m}^{2}$ bei Männern bzw. $<15 \mathrm{~kg} \mathrm{~m}^{2}$ bei Frauen definiert werden [24]. Dies ist aber keine global akzeptierte Definition. Kachexie führt nicht nur zu einer Verschlechterung der Lebensqualität, sondern auch zu einer deutlich reduzierten Belastungskapazität und erhöhten Mortalität [25].

\section{Therapieansätze}

Vermehrte Ernährung und Appetitanreger wurden lange Zeit für kachektische COPD-Patienten propagiert. Hierbei kamen verschiedene Diäten, u.a. hochkalorische Nahrung mit hohem Fettanteil zum Einsatz. Bezüglich der Anwendung werden häufige kleine Mahlzeiten empfohlen, um postprandiale Atemnot und starkes Sättigungsgefühl zu vermeiden [26]. Die Ergebnisse waren jedoch allenfalls mäßig: eine Cochrane Metaanalyse kommt zu dem Ergebnis, dass vermehrte Ernährung keinen messbaren Einfluss auf anthropometrische Messgrößen, Lungenfunktion oder Belastungskapazität hat [27]. Offen bleibt, ob z. B. vermehrte Ernährung im Vorfeld der Manifestation einer Kachexie effektiver wäre. Das Nahrungsergänzungsmittel Kreatin führt zur Erhöhung der fettfreien Masse und angeblich auch zu einer verbesserten Muskelkraft.

Körperliches Training wird als adjunktive Maßnahme angesehen. Körperliche Belastung erhöht jedoch den oxidativen Stress und könnte sich somit im Langzeitverlauf eher schädlich auswirken. Dennoch wird die Belastungskapazität auch bei kachektischen Patienten verbessert. Diese gegensätzlichen Effekte sind gegeneinander abzuwägen.

Ob eine Sauerstofflangzeittherapie mit Korrektur der Hypoxämie einen Einfluss auf Entwicklung oder Umkehr einer Kachexie hat, ist ebenfalls unbekannt. Nach Beginn einer nicht invasiven Beatmung wurde bei kachektischen COPD-Patienten eine deutliche Gewichtszunahme beobachtet, die Körperzusammensetzung jedoch nicht gemessen. Ob der Wirkmechanismus auf einer Reduktion der Atemarbeit beruht, bleibt spekulativ.

Der Einsatz antiinflammatorischer Substanzen wurde in einigen Kurzzeitstudien untersucht, z.B. TNF- $\alpha$ Antikörper (Infliximab) oder das Antioxidans N-acetylcystein. Bisher gibt es keine überzeugenden Beweise einer Effektivität, ebenso wenig durch Blockade der erhöhten sympathomimetischen Aktivität mittels Betablocker oder ACE-Hemmer. Für Omega-3-Fettsäuren gibt es neben theoretischen Vorteilen eine gewisse Evidenz für eine positive Wirkung auf die Muskelfunktion.

Auch anabole Hormone einschl. Wachstumshormonen und Insulin-like growth factor wurden untersucht: Die Ergebnisse sind widersprüchlich: die fettfreie Körpermasse nahm zwar zu, mus- 
kulär wurde jedoch kein Benefit entdeckt, z. B. verbesserte Muskelkraft. Ghrelin, ein Wachstumshormon freisetzendes Peptid, hat dagegen auch zu einer Besserung der Muskelkraft geführt. Alle diese therapeutischen Ansätze beruhen auf physiologischen Rationalen und wurden bisher nur in kleinen Pilotstudien untersucht. Nicht untersucht wurde bisher ein multimodaler Ansatz.

Zusammengefasst gibt es z.Zt. nur eine geringe Evidenz, dass pulmonale Kachexie durch vermehrte enterale Ernährung allein oder durch bestimmte Nahrungszusammensetzung positiv beeinflusst werden kann. Eine Ausnahme stellt die Cystische Fibrose dar - hier ist neben hochkalorischer Ernährung bei exokriner Pankreasinsuffizienz die Substitution von Pankreasenzymen von entscheidender Bedeutung [28]. Ernährung in Kombination mit körperlichem Training erscheint derzeit der aussichtsreichste Ansatz zu sein. Viele Fragen sind z.Zt. noch nicht beantwortet: z.B. warum entwickeln nur einige Patienten eine Kachexie? Welche genetischen oder Umweltfaktoren führen dazu? Was ist die Triggerfunktion? Welche Rolle spielen Komorbiditäten? Welche therapeutischen Ansätze können Kachexie verhindern oder sie zurückbilden? Ein wesentlicher Faktor scheint die Besserung der pulmonalen Funktion zu sein, z.B. führte eine Lungentransplantation bei kachektischen Patienten zu einem deutlichen Gewichtszuwachs, unabhängig von Diagnose, Alter oder Geschlecht. Der Hauptansatz ist daher wohl das Bestreben, die zugrunde liegende pulmonale Erkrankung erfolgreich zu therapieren.

\section{Palliative Versorgung von „Weaning-Versagern“}

In den letzten Jahren ist ein erheblicher Anstieg von Patienten mit Weaningversagen zu verzeichnen [29]. Die Ursachen hierfür sind vielschichtig, die wesentlichen Faktoren sind höheres Lebensalter und zunehmende Polymorbidität bei gleichzeitig verbesserten Behandlungsmöglichkeiten [30]. Vor allem bei Patienten mit fortgeschrittenen Lungen- oder Herzerkrankungen, Patienten mit dialysepflichtigem Nierenversagen sowie deliranten Syndromen und neurokognitivem Defizit ist von einem schwierigen und prolongierten Entwöhnungsprozess auszugehen. Eine weitere große Gruppe stellen Patienten mit Critical Illness Polyneuro- und Myopathie nach schweren Akuterkrankungen wie Sepsis und ARDS dar. Der Anteil solcher langzeitbeatmeten Patienten beträgt zwischen 10 und 20\% aller beatmeten Patienten auf Intensivstationen. Eine kürzlich durchgeführte Erhebung an spezialisierten Beatmungs- und Weaningzentren in Deutschland ergab, dass ca. zwei Drittel dieser Patienten noch erfolgreich entwöhnt werden konnten, von denen wiederum die Hälfte mit einer nicht invasiven Heimbeatmung versorgt werden musste. Ein Fünftel der langzeitbeatmeten Patienten verstarb am Respirator, knapp 13\% der Patienten waren definitiv nicht von der invasiven Beatmung zu entwöhnen [31]. Der Anteil langzeitbeatmeter Patienten mit der Diagnose Weaningversagen ist auf nicht spezialisierten Intensivstationen erheblich größer. Aufgrund der mit dieser Diagnose verbundenen weiteren Versorgungsproblematik sollten diese Patienten spezialisierten Weaningzentren zugewiesen werden, um die Erfolgsrate zu verbessern und die Anzahl von Patienten mit anhaltend invasiver Beatmungspflichtigkeit zu minimieren. Da schwieriges und prolongiertes Weaning sehr häufig mit chronischen Lungenerkrankungen assoziiert ist, haben sich in Deutschland in den letzten Jahren hauptsächlich pneumologische Weaningzentren etabliert.

Patienten, die weiterhin auf eine invasive Beatmung angewiesen sind und eine Fortführung der Beatmung wünschen, müssen heutzutage nicht mehr dauerhaft auf den Intensiv- und Beat- mungsstationen der Kliniken versorgt werden. Die langfristige Betreuung invasiv beatmeter Patienten kann in stationären Pflegeeinrichtungen, in spezialisierten Wohneinheiten oder sogar in der häuslichen Umgebung mit Betreuung durch spezialisierte Pflegedienste erfolgen [32]. Jedoch können und sollten nur spezialisierte Beatmungs- und Weaningzentren aufgrund ihrer langjährigen Erfahrung in der Betreuung nicht entwöhnbarer Patienten entscheiden, ob sich der Patient in einem ausreichend stabilen Zustand befindet, um in eine außerklinische Versorgungsform entlassen zu werden. Die Prognose und das therapeutische Konzept unter Berücksichtigung aller Komorbiditäten tragen hier ebenso zur Entscheidung bei wie die jeweiligen Möglichkeiten der außerklinischen ärztlichen und pflegerischen Betreuung und gegebenenfalls die Verfügbarkeit von Dialyseeinrichtungen. Neben diesen medizinisch-pflegerischen Voraussetzungen beeinflussen die finanziellen Verhältnisse des Patienten, die Ausstattung der eigenen Wohnung, die Entfernung einer Pflegeeinrichtung oder Wohneinheit zum bisherigen sozialen Umfeld und nicht zuletzt der Wunsch bzw. der mutmaßliche Wille des Patienten und die Belastbarkeit des Lebenspartners oder der Familie das Konzept der weiteren Versorgung. Das Überleitungsmanagement invasiv beatmeter Patienten in den außerklinischen Bereich stellt daher hohe Anforderungen an die Kommunikation zwischen allen beteiligten Gruppen: dem klinischen Behandlungsteam, bestehend aus Ärzten, Pflegenden und Sozialarbeitern sowie Seelsorgern und Psychologen, dem Patienten und seinen Angehörigen, dem ambulanten Pflegedienst und dem weiterbehandelnden Arzt, den Medizintechnikfirmen sowie dem medizinischen Dienst und dem Kostenträger. Wenn ein entscheidungsfähiger Patient die Beatmung im Falle eines Weaningversagens nicht mehr fortsetzen möchte, muss im Beatmungszentrum das terminale Weaning mittels kontinuierlicher Reduktion der Atmungsunterstützung bis hin zur finalen Diskonnektion unter ausreichender Sedation eingeleitet werden [33].

Die technischen und personellen Möglichkeiten zur außerklinischen Beatmungspflege sind heute in den meisten Regionen Deutschlands gegeben. Die außerklinische Beatmung ist allerdings nicht als Fortführung einer intensivmedizinischen Behandlung im ambulanten Bereich zu verstehen. Auch bei Patienten mit Weaningversagen kann die ambulante Versorgung eine Betreuung über Jahre bedeuten, sie ist daher den Bedürfnissen des individuellen, selbstbestimmten Lebens und im weiteren Verlauf auch dem würdevollen Sterben verpflichtet. Es ist grundsätzlich eine Versorgungssituation anzustreben, die betroffenen Patienten trotz der unter Umständen 24-stündigen Abhängigkeit von medizinischen Geräten und Pflegepersonen eine neue Lebensperspektive ermöglicht. Zur Reintegration in die Gemeinschaft sind gegebenenfalls weitere technische Hilfsmittel wie Kommunikations- und Mobilitätshilfen erforderlich. Im Vergleich zur Versorgung auf einer Intensivstation kann eine optimal gestaltete außerklinische Beatmungspflege die Lebensqualität verbessern und Betroffenen wieder die Möglichkeit geben, ihr individuelles Potenzial zu entfalten. Dieser Effekt kann das therapeutische Konzept nachhaltig beeinflussen, da einige Patienten unter diesen Bedingungen mittel- bis langfristig doch noch vom Respirator zu entwöhnen sind. Diese Tatsache unterstreicht die Notwendigkeit zur langfristigen Betreuung ambulanter Beatmungspatienten durch die Beatmungs- und Weaningzentren.

Die außerklinische invasive Beatmung muss als Behandlungsmaßnahme kritisch hinterfragt werden, wenn sie weder zu einer für den Patienten annehmbaren Lebensqualität führt oder einen Sterbeprozess verlängert bzw. gar erschwert. Allerdings haben 
Tab. 2 OPS Kode 2009:8-982 Palliativmedizinische Komplexbehandlung.

Hinw.: Mindestmerkmale
Durchführung eines standardisierten palliativmedizinischen Basis-
assessments (PBA) zu Beginn der Behandlung
Aktive, ganzheitliche Behandlung zur Symptomkontrolle und psycho-
sozialen Stabilisierung ohne kurative Intention und im Allgemeinen
ohne Beeinflussung der Grunderkrankung von Patienten mit einer
progredienten, fortgeschrittenen Erkrankung und begrenzter Lebens-
erwartung unter Einbeziehung ihrer Angehörigen und unter Leitung
eines Facharztes mit der Zusatzweiterbildung Palliativmedizin
Aktivierend- oder begleitend-therapeutische Pflege durch besonders
in diesem Bereich geschultes Pflegepersonal
Erstellung und Dokumentation eines individuellen Behandlungsplans
bei Aufnahme
Wöchentliche multidisziplinäre Teambesprechung mit wochen-
bezogener Dokumentation bisheriger Behandlungsergebnisse und
weiterer Behandlungsziele
Einsatz von mindestens zwei der folgenden Therapiebereiche:
Sozialarbeit/Sozialpädagogik, Psychologie, Physiotherapie, künstle-
rische Therapie (Kunst- und Musiktherapie), Entspannungstherapie,
Patienten-, Angehörigen- und/oder Familiengespräche mit insgesamt
mindestens 6 Stunden pro Patient und Woche in patientenbezogenen
unterschiedlichen Kombinationen (Die Patienten-, Angehörigen-
und/oder Familiengespräche können von allen Berufsgruppen des
Behandlungsteams durchgeführt werden.)
8 - 982.0 Bis zu 6 Behandlungstage
8 - 982.1 Mindestens 7 bis höchstens 13 Behandlungstage
8 - 982.2 Mindestens 14 bis höchstens 20 Behandlungstage
8 - 982.3 Mindestens 21 Behandlungstage

auch invasiv beatmete Patienten im Sterbeprozess das Recht, der häuslichen Versorgung den Vorzug zu geben. Beatmungs- und Weaningzentren müssen daher auch in diesen Situationen bemüht sein, eine optimale Versorgungssituation zu schaffen. Ebenso sollten sich die Zentren verpflichtet fühlen, ambulant versorgte Patienten mit dem Wunsch nach Beatmungsabbruch zu begleiten. Der Wunsch nach Therapieabbruch kann sich aufgrund des Erkrankungsfortschritts ergeben, darf aber nicht durch eine für den Patienten unzumutbare Versorgungssituation begründet sein. In diesen Situationen ist das gesamte ambulante und klinische Versorgungsnetzwerk gefordert, Lösungsansätze zu finden, die Versorgung durch Symptomlinderung zu verbessern oder den ärztlich geleiteten Beatmungsabbruch einzuleiten.

\section{Versorgungsstrukturen \\ $\nabla$}

\section{Stationäre palliative Versorgung: Mindestanforderun-} gen, Strukturen, Finanzierung

„Fallpauschalen im Krankenhaus - Das Ende der Barmherzigkeit“ titelte das Deutsche Ärzteblatt bereits im Dezember 2001, also ein Jahr vor der endgültigen Einführung der Fallpauschalen, im folgenden DRG (Diagnosis Related groups) genannt. Am 1. Januar 2000 ist im Rahmen des GKV-Gesundheitsreformgesetzes ein neuer $\S 17 \mathrm{~b}$ des Krankenhausfinanzierungsgesetzes (KHG) in Kraft getreten. Damit wurde ab 2003 die Einführung eines pauschalisierten Abrechnungssystems in die Wege geleitet. Nach 2 budgetneutralen Jahren 2003 und 2004 begann ab 2005 die sogenannte Konvergenzphase, in der schrittweise eine Angleichung der Krankenhausentgelte auf ein einheitliches Niveau in den jeweiligen Bundesländern bis 2009 erreicht wurde.

Im ersten DRG-Jahr 2003 war die Palliativtherapie in keiner Weise abgebildet. Dies war bereits in frühen Publikationen als Man-
Tab. 3 Höhe des Zusatzentgeltes 60.

\section{Palliativmedizinische Komplexbehandlung mehr als 6 Tage, OPS Kode: 8-982.1-8-982.3}

2007: EUR 1101,46

2008: EUR 1365,89

2009: EUR 1347,43

gel angesehen worden [34]. Sonderentgelte waren im ersten DRG System 2003 nicht vorgesehen. Aufgrund fehlender Kennzeichnungsmerkmale für Palliativpatienten im Anfangsjahr der DRG Einführung war eine Identifizierung dieser Patienten nicht möglich.

2004 wurde dann erstmals eine Ausnahmeregelung als Alternative zur nun verbindlichen DRG Abrechnung für sogenannte „besondere Einrichtungen“ geschaffen. Für die als „besondere Einrichtungen" anerkannten Bereiche konnten damit tagesgleiche Entgelte aufgrund nachgewiesener Kostenstrukturen mit den Kostenträgern vereinbart werden. Hier wurde unter anderem auch für bereits etablierte Palliativeinheiten eine Möglichkeit zur kostenadäquaten Abrechnung geschaffen. Diese Möglichkeit einer Ausnahmeregelung zur DRG Abrechnung wurde bisher von Jahr zu Jahr fortgeschrieben. Für 2009 war von den Kostenträgern bereits eine Herausnahme von Palliativstationen aus den „besonderen Einrichtungen“ gefordert worden. Dies fand jedoch innerhalb der Selbstverwaltung keinen Konsens. Stattdessen wurde das InEK aufgefordert nochmals die Kostenstrukturen der Palliativbehandlung sowie die Abbildung im DRG System für 2010 zu überprüfen. Eine Aufnahme von neu eingerichteten Bereichen in diese Regelung wird von den Kostenträgen mit Hinweis auf die Weiterentwicklung des Systems nicht akzeptiert. Durch die Ausdifferenzierung des DRG Systems in den letzten Jahren ist aber zukünftig mit einem Auslaufen dieser Regelung zu rechnen.

Auf Initiative der Fachgesellschaften wurde dann für das Jahr 2005 ein OPS Kode ( $\bullet$ Tab. 2 ) geschaffen, um die Palliativtherapie spezifisch abbilden zu können. Dieser OPS Kode beschreibt genaue Strukturmerkmale sowie individuell bei den jeweiligen Patienten zu erfüllende Zeitvorgaben ( $\bullet$ Tab. 2).

Diese Prozeduren standen für die Kalkulation des DRG Systems 2007 erstmalig für eine Analyse zur Verfügung. Dabei zeigte sich, dass diese Leistung die Voraussetzungen zur Etablierung eines Zusatzentgelts wie beispielsweise Streuung über mehrere DRG's oder definierbare Leistung mit eindeutigem Identifikations- und Abrechnungsmerkmal erfüllt. Daher wurde mit dem ZE60 Palliativmedizinische Komplexbehandlung ein neues, bewertetes $\mathrm{Zu}-$ satzentgelt in Anlage 2 bzw. 5 der Fallpauschalenvereinbarung 2007 aufgenommen.

Die Höhe des Zusatzentgeldes wird anhand der jährlichen Datenlieferungen neu kalkuliert, hierdurch ergeben sich die Schwankungen in der Höhe des Erlöses über die Jahre ( $\bullet$ Tab. 3).

Zur Abrechnung einer palliativmedizinischen Komplexbehandlung ist explizit nicht eine Palliativstation gefordert, sondern lediglich die Erfüllung der Strukturvorgaben. Ab 2009 sind weitere Mindestvoraussetzungen hinzugekommen, so insbesondere eine Basisdokumentation bei Beginn der palliativmedizinischen Versorgung.

Die Einhaltung der Strukturvorgaben ist den Kostenträgern mittels eines detaillierten Fragebogens nachzuweisen, wobei die entsprechenden Qualifikationsträger namentlich aufzuführen sind. 
Weiterhin muss die Erfüllung der Zeitvorgaben (Einsatz von mindestens zwei der folgenden Therapiebereiche: Sozialarbeit/Sozialpädagogik, Psychologie, Physiotherapie, künstlerische Therapie (Kunst- und Musiktherapie), Entspannungstherapie, Patienten-, Angehörigen- und/oder Familiengespräche mit insgesamt mindestens 6 Stunden pro Patient und Woche in patientenbezogenen unterschiedlichen Kombinationen) patientenindividuell im Einzelfall dokumentiert werden. Bei MDK-Prüfungen wird ein besonderes Augenmerk auf den Nachweis der regelmäßigen wöchentlichen Teambesprechungen mit Dokumentation von Teilnehmern sowie der individuellen Patientenbesprechung gelegt. Die Art der Dokumentation der einzelnen Vorgaben ist nicht festgeschrieben, sie muss jedoch gut nachvollziehbar sein.

Insgesamt steht mit dem Zusatzentgeld „Palliativmedizinische Versorgung“ (ZE60) eine Abbildung des Mehraufwandes der stationären palliativmedizinischen Betreuung zur Verfügung. Die Realisierung dieses Erlöses ist jedoch an hohe strukturelle Anforderungen und penible Dokumentation gebunden.

Institute

1 Zentrum für Pneumologie und Thoraxchirurgie, Krankenhaus Großhansdorf (Direktor: H. Magnussen)

2 Department of Palliative Care, Policy \& Rehabilitation, King's College London, England (I.J. Higginson)

3 Innere Medizin/Onkologie, Thoraxklinik am Universitätsklinikum Heidelberg (M. Thomas)

${ }^{4}$ Abteilung für Pneumologie, Asklepios Klinik Hamburg-Harburg (G. Wiest)

${ }^{5}$ Krankenhaus Martha Maria, Halle-Dölau (W. Schütte)

${ }^{6}$ Medizinische Klinik m. S. Infektiologie und Pneumologie, Campus Mitte,

Charité, Berlin (N. Suttorp)

7 Fehmarner Str. 5, 13353 Berlin

8 Asklepios Fachkliniken München-Gauting (O. Karg)

9 Serrahnstr. 3, 21029 Hamburg

\section{Literatur}

1 Lanken PN, Terry PB, Delisser HM et al. An official American Thoracic Society clinical policy statement: palliative care for patients with respiratory diseases and critical illnesses. Am J Respir Crit Care Med 2008; 177: $912-927$

2 Nava S, Sturani C, Hartl S et al. End-of-life decision-making in respiratory intermediate care units: a European survey. Eur Respir J 2007; 30: $156-164$

3 Gregor A, Thomson CS, Brewster DH et al. Management and survival of patients with lung cancer in Scotland diagnosed in 1995: results of a national population based study. Thorax 2001; 56: $212-217$

4 Husebo S;Klaschik E. Palliativmedizin. Heidelberg: Springer, 2006

5 Scano G, Stendardi L, Grazzini M. Understanding dyspnoea by its language. Eur Resp J 2005; 25: 380 - 385

6 Bruera E, Sweeney C, Willey J et al. A randomized controlled trial of supplemental oxygen versus air in cancer patients with dyspnea. Palliative Medicine 2003; 17: 659-663

7 Bausewein C, Booth S, Gysels M et al. Non-pharmacological interventions for breathlessness in advanced stages of malignant and non-malignant diseases. Cochrane Database Syst Rev 2008; January 1: CD005623

8 Jennings AL, Davies AN, Higgins JP et al. Opioids for the palliation of breathlessness in terminal illness. Cochrane Database Syst Rev 2001: CD002066

9 Ripamonti C, Fulfaro F, Bruera E. Dyspnoea in patients with advanced cancer: incidence, causes and treatments. Cancer Treat Rev 1998; 24 : $69-80$

10 Abernethy AP, Uronis HE, Wheeler JL et al. Pharmacological management of breathlessness in advanced disease. Progress in Palliative Care 2008; 16: $15-20$
11 Bausewein C, Rémi C, Twycross R et al. Arzneimitteltherapie in der Palliativmedizin. München, Jena: Urban und Fischer, 2005: 133-206

12 Flieder M, Jansen JP. Praxishandbuch Palliativpflege und Schmerzmanagement. Merching: Forum, 2007

13 Husebo S, Klaschik E. Palliativmedizin. Heidelberg: Springer, 2006: 203-307

14 Aulbert E, Nauck F, Radbruch L. Lehrbuch der Palliativmedizin. Stuttgart: Schattauer, 2007: 148-269

15 Minton O, Richardson A, Sharpe M et al. A systematic review and metaanalysis of the pharmacological treatment of cancer-related fatigue. J Natl Cancer Inst 2008; 100(16): 1155-1166

16 Morrow GR, Jean-Pierre P, Roscoe JA et al. A phase III randomized, placebo-controlled, double-blind trial of a eugeroic agent in 642 cancer patients reporting fatigue during chemotherapy: a URCC CCOP study (abstract). J Clin Oncol 2008; 26: 504s

17 Henke M, Laszig R, Rube C et al. Erythropoietin to treat head and neck cancer patients with anaemia undergoing radiotherapy: randomised, double-blind, placebo-controlled trial. Lancet 2003; 362: 1255-1260

18 Thorsen L, Skovlund E, Stromme SB et al. : Effectiveness of physical activity on cardiorespiratory fitness and health-related quality of life in young and middle-aged cancer patients shortly after chemotherapy. J Clin Oncol 2005; 23: 2378-2388

19 Magnussen H, Kirsten AM, Köhler D et al. Leitlinien zur Langzeit-Sauerstofftherapie. Pneumologie 2008; 62: 748-756

20 Windisch $W$, Criée CP. Lebensqualität bei Patienten mit häuslicher Beatmung. Pneumologie 2006; 60: 539-546

21 Windisch $W$. Quality of life in home mechanical ventilation study group. Impact of home mechanical ventilation on health-related quality of life. Eur Respir J 2008; 32: 1328 - 1336

22 Garrod R, Mikelsons C, Paul EA et al. Randomized controlled trial of domiciliary noninvasive positive pressure ventilation and physical training in severe chronic obstructive pulmonary disease. Am J Respir Crit Care Med 2000; 162: 1335 - 1341

23 Leger P, Bedicam JM, Cornette A et al. Nasal intermittent positive pressure ventilation. Long-term follow-up in patients with severe chronic respiratory insufficiency. Chest 1994; 105: 100-105

24 Schols AMWJ, Broekhuizen R, Weling-Scheepers CA et al. Body composition and mortality in chronic obstructive disease. Am J Clin Nutr 2005; 82: $53-59$

25 Schols AMWJ. Pulmonary cachexia. Inter J Cardiol 2002; 85: 101 - 110

26 Anker SD, John M, Pedersen PU et al. ESPEN Guidelines on Enteral Nutrition: Cardiology and Pulmonology. Clin Nutr 2006; 25: 311-316

27 Ferreira I, Brooks D, Lacasse Y et al. Nutritional supplementation for stable chronic obstructive pulmonary disease. Cochrane Database Syst Rev 2005: CD000998

28 Posselt HG, Smaczny C, Stern M et al. Leitlinie Mukoviszidose (Cystische Fibrose): Ernährung und exokrine Pankreasinsuffizienz. www.awmfonline.de; Stand: 19.10.2005

29 Cox CE, Carson SS, Holmes GM et al. Increase in tracheostomy for prolonged mechanical ventilation in North Carolina, 1993-2002. Crit Care Med 2004; 32: 2219-2226

30 MacIntyre NR, Epstein SK, Carson S et al. National association for medical direction of respiratory Care. Management of patients requiring prolonged mechanical ventilation: report of a NAMDRC consensus conference. Chest 2005; 128: 3937-3954

31 Schönhofer B, Berndt C, Achtzehn U et al. Weaning from mechanical ventilation. Dtsch Med Wochenschr 2008; 133: 700-704

32 Schönhofer B, Euteneuer S, Nava S et al. Survival of mechanically ventilated patients admitted to a specialised weaning centre. Intensive Care Med 2002; 28: 908-916

33 Ankrom M, Zelesnick L, Barofsky I 3rd. et al. Elective discontinuation of life-sustaining mechanical ventilation on a chronic ventilator unit. J Am Geriatr Soc 2001; 491: 549-554

34 Roeder N, Klaschik E, Cremer M et al. DRGs in der Palliativmedizin: Ist die palliativmedizinische Begleitung Schwerstkranker pauschalierbar? Das Krankenhaus 2002; 12: 1000-1004 